\title{
Cognitive Dissonance in Iranian Multiple Sclerosis Patients
}

\author{
Atefeh Ashrafi Birgani* and Elham Foroozandeh \\ Department of Psychology, Islamic Azad University, Iran
}

*Corresponding author: Atefeh Ashrafi Birgani, Department of Psychology, Naein Branch, Islamic Azad University, Naein, Iran.

To Cite This Article: Atefeh Ashrafi Birgani. Cognitive Dissonance in Iranian Multiple Sclerosis Patients. Am J Biomed Sci \& Res. 2019 - 4(3). AJBSR.MS.ID.000789. DOI: 10.34297/AJBSR.2019.04.000789

Received: : July 07, 2019 | Published: July 26, 2019

\begin{abstract}
Multiple Sclerosis is a chronic disease characterized by loss of myelin in neurons.

Objective: The aim of this study was to compare the cognitive dissonance of multiple sclerosis (MS) patients with healthy people.

Methodology: MS participants were selected using non-probability sampling method from Iranian patients and healthy participants were paired with MS sample in education, age and illness stage. They were asked to complete dissonance arousal and reduction questionnaire (Harmon-Jones;2008).
\end{abstract}

Discussion: The results showed that the average score of MS patients was significantly higher than non-clinical subjects (p<0.05). It can be stated that one of the basic reasons of dissonance cognition in MS referred to probably impairment of memory, learning and cognitive functions.

Keywords: Cognitive dissonance; Multiple sclerosis; Cognitive process

Abbrevations: MS: Multiple Sclerosis

\section{Introduction}

Multiple Sclerosis (MS) is a progressive neurologic disease that cause disabilities in individual and social abilities and is seen in people between 18-45years [1]. Neuronal loss is a hallmark of disease progression and disability in multiple sclerosis (MS) [2]. The clinical course of multiple sclerosis (MS) is highly variable, ranging from rapidly reversible episodes of impairment to severe disability within months after disease onset. Focal inflammation, chronic diffuse neuronal damage, and failure of repair or compensation all contribute to the development of permanent disability [3]. In the early 1900s, only a few cases of multiple sclerosis (MS) were reported, which quickly became a common occurrence for admission to neurological wards. Today, MS accounts over 2.5 million affected individuals with an estimated cost of US $\$ 2-3$ billion per annum. The distribution of MS varies according to geographic location. For example, the further north or south from the equator the higher the prevalence of MS; countries that lie on the equator have extremely low prevalence compared to Scotland, Norway, and Canada. The prevalence of MS has increased progressively over time with 30/100,000 diagnosed in 2008 to 33/100,000 diagnosed in 2013 globally. In fact, in a Norwegian cohort over 53 years
(1961-2014), the prevalence increased from 20 to 203/100,000 and the incidence increased from 1.9 to $8 / 100,000$ It is possible that the increase in prevalence is due to improved diagnostic procedures and reporting and changes in lifestyle (lack of vitamin D and increased smoking) [4]. There are now 16 US Food and Drug Administration (FDA)-approved disease-modifying therapies for MS as well as a cohort of other agents commonly used in practice when conventional therapies prove inadequate [5]. Virtually all MS medications now in use target the immune system and prevent tissue damage by modulating neuroinflammatory processes. Although current therapies such as commonly prescribed diseasemodifying medications decrease the relapse rate in relapsingremitting MS (RRMS), the prevention of long-term accumulation of deficits remains a challenge. Medications used for progressive forms of MS also have limited efficacy [6]. Impairments in higher level cognitive processing, such as new learning and memory, are common in Multiple Sclerosis (MS) and negatively impact multiple aspects of everyday life, including occupational and social functioning. The previous studies showed that the memory problems that patients with MS experience are in learning new 
information, not retrieving previously learned information from memory storage. Patients have trouble remembering things such as tasks they need to do in a given day, an address or a list of items. They may have difficulty learning and remembering how to do a new task. These are all everyday applications of memory abilities. It is this cognitive profile that makes persons with MS particularly appropriate for interventions targeting specific cognitive functions, such as new learning and memory or processing speed. If they are not showing a general cognitive decline, the cognitive decline is most often specific and subtle [7]. Neuropsychological anomalies related to MS can be divided into two groups: mood and behaviour disorders (depression, euphoria, abnormal crying and laughing) and cognition disorders (Disturbance in attention, Fast information processing, focus, learning, Memory) [8].

Dissonance cognition is a state of tension which achieved through having two simultaneous cognitive process (thought, attitudes, beliefs) that are incongruent, psychologically. Inconsistency in some combination of cognition and behaviour causes an uncomfortable arousal state that labelled "dissonance cognition". The basic assumption is that dissonance cognition leads to Irregularity, discomfort and tension. In order to reduce the inconsistency, the person tries to decrease inconsistent elements or values of them. A person who experiences internal inconsistency tends to become psychologically uncomfortable, and so is motivated to reduce the cognitive dissonance. When there is a consistent cognition, action performed effectively without any confliction but when a person faces with phenomena cause to inconsistency, conflicting cognitions appears. Then the way of believing or behaviour changed. Therefore, inconsistency as an arousal state is based on elimination of transient negative emotions in the way that thoughts and behaviours processed effectively, without any conflictions.

MS patients experienced unexpected and descending trend of health, changing in social and friendship relations and support and should confront with them [9]. In this study the question is that if the MS patients are statistically different in their cognitive dissonance with health people?

\section{Subjects and Methods}

In order to gathering information 50 patients who referred to MS centre in Isfahan, Iran and 50 healthy participants were selected. The entrance criteria include the range of 18 to 55 years old and educated degree of third grade. In order to gathering information and measuring variables, dissonance arousal and reduction questionnaire (DARQ) has been used. Dissonance arousal and reduction questionnaire has provided by Harmon- Jones (2008) and translated to Persian by Zandi [10]. Validity and Reliability of DARQ was approved in Iranian Sample.

\section{Results}

\begin{tabular}{|c|c|c|c|c|c|c|}
\hline \multicolumn{7}{|c|}{ Table 1: Descriptive statistics of dissonance cognition scores } \\
\hline Variable & Group & N & mean & SD & Max & Min \\
\hline Dissonance & MS patients & 50 & 48.22 & 5.48 & 59 & 37 \\
\hline Cognition & Non-patients & 50 & 37.98 & 8.11 & 65 & 24 \\
\hline
\end{tabular}

Results show the mean score of dissonance cognition is higher in MS patients (Table 1).

For comparison of mean scores of dissonance cognition in two groups, ANOVA was calculated. As stated in Table 2, difference in dissonance cognition between healthy and unhealthy people is statistically significant $(\mathrm{p}>0.05)$.

Table 2: ANOVA for comparison between dissonance cognition in two groups

\begin{tabular}{|c|c|c|c|c|}
\hline Sum of squares & df & Mean of squares & F & Sig. \\
\hline 2621.44 & 1 & 2621.44 & 54.665 & 0.001 \\
\hline
\end{tabular}

\section{Discussion}

Difference between mean scores of dissonance cognition of healthy and MS patients is statistically significant. The theory of cognitive dissonance is based on the principle that individuals seek to reconcile their expectations and their reality. The four ways to reduce mental tension in inconsistency are:

i. Change of Behaviour or Cognition,

ii. Justifying behaviour or cognition through a change in conflicting cognition,

iii. Justifying behaviour or cognition by adding a new cognition,

iv. Denying or ignoring any information about conflicting knowledge.

The basic assumption is that dissonance cognition leads to Irregularity, discomfort and tension. More discrepancy leads to more stressful situation and more tries to reduce the inconsistency. In order to reduce the inconsistency, the person tries to decrease inconsistent elements or values of them. It is believed when emotional and cognitive information processed and evaluated wrongly, emotions and cognitions perform incorrectly, and it is so probable to use dissonance cognition in a stressful condition. As a result of this study, it can be stated that one of the basic reasons of dissonance cognition in patient who suffered from M.S, referred to impairment of cognitive function (memory, learning, information processing and etc.) [11]. Since that Multiple Sclerosis inflicts tensions, it can be reduced dissonance cognition in stressful situations. People in inconsistence conditions experienced extreme stress and they cannot reduce negative emotions raised because of dissonance cognition phenomenon. Also, they cannot change their attitudes and experience irritation in several conditions such as making important decisions.

In this study non-probability sampling was used and a small MS patients group. So, we suggest for future studies that using probability sampling and greater sample, more details can be discovered about cognitive process in MS patients.

\section{Conclusion}

Dissonance cognition is an experience that leads to Irregularity and tension, and to control and reduce the inconsistency, MS patient tries to decrease inconsistent elements. When emotional 
and cognitive information processed and evaluated wrongly, emotions and cognitions perform incorrectly, and it is so probable to use dissonance cognition in a stressful condition. It can be stated that one of the basic reasons of dissonance cognition in patient who suffered from M.S, referred to probably impairment of memory, learning and cognitive functions.

\section{References}

1. Coleman CI, Sidovar MF, Roberts MS, Kohn C (2013) Impact of Mobility Impairment on Indirect Costs and Health- Related Quality of Life in Multiple Sclerosis. PLoS One 8(1): e547-566.

2. J Kuhle, C Barro, A.H Brachat, M A Valentin, R Hillenbrand, et al. (2016) Blood neurofilament light chain levels are elevated in multiple sclerosis and correlate with disease activity. Paper presented at: 32nd Congress of the European Committee for Treatment and Research in Multiple Sclerosis, London, United Kingdom.

3. Romme Christensen J, Börnsen L, Khademi M, Olsson T, Jensen PE, et al. (2013) CSF inflammation and axonal damage are increased and correlate in progressive multiple sclerosis. Mult Scler 19(7): 877-884.

4. Dargahi N, Katsara M, Tselios T, Androutsou ME, De Courten M, et al. (2017) Multiple Sclerosis: Immunopathology and Treatment Update. Brain Sci 7(7): pii E78.
5. Teresa C Frohman, Shin C Beh, Eric J Kildebeck, Ram Narayan, Katherine Treadaway, et al. (2016) Neurotherapeutic Strategies for Multiple Sclerosis. Neurologic Clinics 34(3): 483-523.

6. Farjam M, Zhang GX, Ciric B, Rostami A (2015) Emerging immunopharmacological targets in multiple sclerosis. J Neurol Sci 358(1-2): 22-30.

7. Plantone D, Renna R, Sbardella E, Koudriavtseva T (2015) Concurrence of multiple sclerosis and brain tumors. Front. Neurol 6: 40.

8. Diamond BJ, Johnson SK, Kaufman M, Graves L (2008) Relationships between information processing, depression, fatigue and Cognition in multiple sclerosis. Arch clin Neuropsychology 23(2): 189-199.

9. Smeltzer SC, Bare BG, Hinkle JL, Cheever KH (2009) Brunner and Suddarth's Textbook of Medical Surgical Nursing: In One Volume: Lippincott Williams \& Wilkins, United States.

10.Zandi A, Safariniya M, Shaghaghi F (2010) Standardized questionnaire and dissonance arousal (DARQ) students of payam nor University of Tehran. Ministry of science, Research and Tecnology, Payam Nor University of Tehran, Tehran.

11. Vaillant GE (1992) Ego Mechanisms of Defense: A Guide for Clinicians and Researchers, American Psychiatric Press, Washington, DC, USA. 